\title{
RESEARCH HIGHLIGHT Making ends meet in class switch recombination
}

\author{
Lizhen $\mathrm{Wu}^{1}$ and David G. Schatz (D) \\ Cell Research (2020) 30:711-712; https://doi.org/10.1038/s41422-020-0342-5
}

\begin{abstract}
Liu et al. revealed that ERCC6L2 is a DNA double strand break repair factor that both enhances the efficiency of immunoglobulin gene class switch recombination and dictates the proper orientation of end joining. ERCC6L2 rapidly localizes to DNA breaks and is necessary for efficient recruitment of other repair factors, suggesting an integral and early role in the complex process that allows for the expression of antibodies with different effector functions.
\end{abstract}

To promote comprehensive and effective adaptive immunity, lymphocytes first establish a large repertoire of immunoglobulins (Ig) and T cell receptors by V(D)J recombination. Immunoglobulins are further diversified by somatic hypermutation (SHM) to enhance antigen recognition and class switch recombination (CSR) to alter effector domains. During CSR, AID initiates double strand breaks (DSBs) in S-regions located $5^{\prime}$ of the coding constant regions defining the antibody classes in the $\mathrm{lgH}$ locus. DSBs are initially sensed by the MRN complex which activates ATM, followed by the recruitment of 53BP1 and other downstream factors. DNA breaks are resolved by the well-characterized c-NHEJ pathway and the less defined alternative end-joining (Alt-EJ) pathway. c-NHEJ events involve limited DNA end resection and few or no sequence microhomologies ( $\mathrm{MHs}$ ), and are mediated by a cascade of factors including Ku, DNAPKcs, Artemis, XRCC4, XLF and LIG4. ${ }^{1}$ Additional factors that regulate NHEJ have been identified recently, such as MRI/CYREN. ${ }^{2,3}$ The Alt-EJ pathway in contrast involves more DNA end resection and often joins DNA ends sharing a few base pairs of $\mathrm{MH} .^{1}$ Notably, DSBs in $\mathrm{S}$ region have to be stitched together in one particular orientation to yield productive recombination. Intra-S rejoining or an inactivating inversion would generate a nonfunctional product. It has been shown that deletional recombination represents $90 \%$ of DSB repair during CSR, an overrepresentation compared to random DNA breaks, which are typically repaired by deletion and by inversion in equal proportions. ${ }^{4}$ The mechanism by which AIDinitiated CSR is biased to the productive deletional orientation is largely unknown. In a recent paper published in Cell Research, Liu et al. make a significant advance in this area by identifying ERCC6L2 as a new factor that promotes DSB end joining and orientation-specific CSR. ${ }^{5}$

Several lines of evidence have established a working model for CSR involving loop extrusion of DNA by the cohesin complex to bring the two recombining S-regions into proximity, with cohesin contributing to the long-range contacts between cis DNA elements such as enhancers and promoters required for CSR, and helping to establish the S-S synapse status ${ }^{6-8}$ (Fig. 1a). 53BP1 promotes orientation-biased recombination, perhaps as a consequence of its multiple functions in CSR including preventing excess DNA end resection to facilitate NHEJ, promoting synapsis by tethering unjoined ends, and ensuring that $S \mu$ incurs DSBs ahead of other acceptor S-regions, which has been proposed to be important for efficient CSR. ${ }^{4,9}$ Lig4 deficiency results in more inversional joining, possibly due to escaped broken ends joined in a fashion similar to that occurring in spontaneous DNA breaks. ${ }^{10}$ Liu et al. now add ERCC6L2 to this small group of factors that repress inversional joining during $\mathrm{CSR}$, revealing that it plays a particularly central role in the process. ${ }^{5}$

Using a CRISPR-knockout screen on genes implicated in DNA repair and different classes of DNA damage-inducing compounds, Liu et al. demonstrated that Baz1b and Ercc6/2 clustered with core NHEJ factors like $K u$, Lig4 and DNAPKCs, implying that they could be novel regulators of NHEJ. The authors then focused on ERCC6L2 because Ercc6/2 mutations have been implicated in inherited bone marrow failure (BMF) syndromes, ${ }^{11}$ as is the case for other NHEJ factors. Ercc6/2-deficient cells are selectively hypersensitive to DSB damage, further implicating ERCC6L2 in DSB repair and potentially NHEJ. Ercc6/2 deficiency results in a twofold decrease in CSR in both the activated $\mathrm{CH} 12$ B lymphoma cell line and splenic B cells stimulated to induce CSR, but does not compromise SHM in geminal centers of immunized mice.

Liu et al. then performed a series of elegant experiments to characterize the mechanisms by which ERCC6L2 functions in CSR. Using laser-microirradiation, they demonstrated that ERCC6L2 is recruited to sites of damage within seconds, similar to NHEJ factors like Ku70 and XLF. ERCC6L2 is comprised of a Tudor domain, an ATPase/Helicase domain, and an extensive C-terminal region containing a conserved HEBO domain (Fig. 1b). Complementation experiments in Ercc6/2 ${ }^{-/-}$cells revealed that the helicase catalytic domain is required for ERCC6L2 to promote CSR. Notably, an ERCC6L2 catalytic-dead mutant and mutants containing only $\mathrm{N}$-terminal fragments due to frameshift have been identified in BMF patients. Liu et al. also demonstrated that the ERCC6L2 C-terminal region is sufficient for nuclear localization and recruitment to DNA damage sites, and is required but not sufficient for ERCC6L2 function in CSR. Furthermore, the ERCC6L2 C-terminal region was found to interact with MRI/CYREN, which was confirmed by pull-downs of ERCC6L2-HA (hemagglutinin tag) in CSR-activated B cells isolated from Ercc6/2-HA knock-in mice. Data from Liu et al. and others suggest that ERCC6L2 interacts with additional NHEJ factors. Interestingly, Liu et al. showed that the recruitment of several NHEJ subunits, including XRCC4, LIG4 and MRI, to sites of DNA damage is compromised in the absence of ERCC6L2, whereas the recruitment of ERCC6L2 to damage sites is independent of NHEJ factors. Their data suggest a model in which ERCC6L2 promotes CSR in part by facilitating the recruitment of NHEJ components to damage sites to complete end-joining.

Finally, by taking advantage of high-throughput genome-wide translocation sequencing (HTGTS), the authors examined end

'Department of immunobiology, Yale School of Medicine, New Haven, CT 06519, USA

Correspondence: David G. Schatz (david.schatz@yale.edu)

Published online: 25 May 2020 
712

a

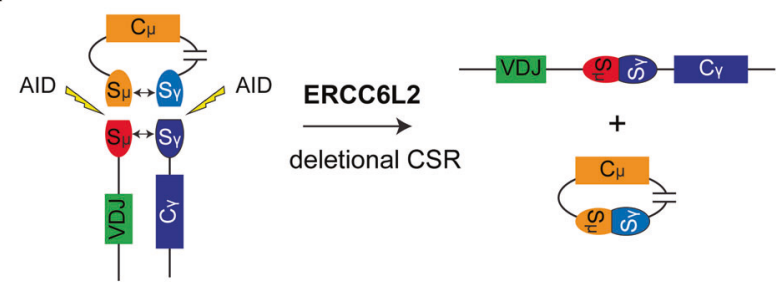

b

ERCC6L2

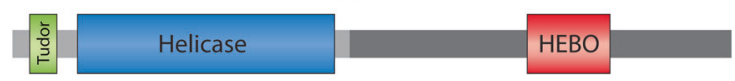

Fig. 1 ERCC6L2 promotes deletional CSR. a AID initiates DSBs in S regions of $\operatorname{lgH}$. Loop extrusion is proposed to bring donor and acceptor $S$ regions into close proximity. Deletional recombination generates a functional product while the intra DNA is looped out. ERCC6L2 promotes this productive pathway of deletional CSR. b Schematic diagram of the domain structure of ERCC6L2.

resection, $\mathrm{MH}$ usage and orientation of DNA end joining during CSR in $\mathrm{ErCC}_{6 / 2^{-1-}} \mathrm{B}$ cells. They observed a remarkable decrease in deletional recombination (55\% in Ercc $6 / 2^{-1-}$ cells vs > $90 \%$ in WT cells), a reduction comparable to that in $53 \mathrm{bp} 1^{-/-}$cells $(60 \%)$. ATM-dependent DSB response factors including 53BP1 limit end resection, thereby promoting deletional $\mathrm{CSR}^{4} \mathrm{~A}$ mild increase in end resection and a slight increase in longer $\mathrm{MH}$ usage in Ercc6/2 ${ }^{-1-}$ cells were observed. However, the increases are much weaker than that in 53BP1-, ATM- or XLF-deficient cells. The modest increases cannot explain the striking loss of deletional recombination, arguing that the mechanism of action of ERCC6L2 differs from that of ATM-dependent DSB response factors. Analysis of B cells expressing catalytic-dead ERCC6L2 indicated that its predicted helicase activity is required for deletional repair, as the mutant shows a twofold CSR reduction and a drop in deletional recombination to $57 \%$.

The findings of Liu et al. strongly implicate ERCC6L2 in NHEJ and orientation-specific CSR and raise a number of interesting questions, foremost among them being how the action of ERCC6L2 is coordinated with that of other factors to enforce deletional joining. One possibility is that ERCC6L2 facilitates ligation complex loading to ensure rapid completion of end joining by directly removing nucleosomes; ERCC 6 family members have been proposed to function as nucleosome remodelers. ${ }^{12}$ It is thus possible that ERCC6L2 functions as a chromatin remodeler, helping to shape chromatin architecture during synapsis to promote deletional CSR.

\section{ADDITIONAL INFORMATION}

Competing interests: The authors declare no competing interests.

\section{REFERENCES}

1. Methot, S. P. \& Di Noia, J. M. Adv. Immunol. 133, 37-87 (2017).

2. Arnoult, N. et al. Nature 549, 548-552 (2017).

3. Hung, P. J. et al. Mol. Cell 71, 332-342 (2018).

4. Dong, J. et al. Nature 525, 134-139 (2015).

5. Liu, X. et al. Cell Res. https://doi.org/10.1038/s41422-020-0328-3 (2020).

6. Zhang, X. et al. Nature 575, 385-389 (2019).

7. Wuerffel, R. et al. Immunity 27, 711-722 (2007).

8. Thomas-Claudepierre, A. S. et al. J. Exp. Med. 210, 2495-2502 (2013).

9. Rocha, P. P. et al. Cell Rep. 16, 48-55 (2016).

10. Panchakshari, R. A. et al. Proc. Natl. Acad. Sci. USA 115, 762-767 (2018).

11. Tummala, H. et al. Am. J. Hum. Genet. 94, 246-256 (2014).

12. Ke, Y. W. et al. EMBO J. 30, 3309-3321 (2011). 